See http://www.oxfordjournals.org/our_journals/ndtplus/

\title{
Combined nephrectomy and pre-emptive renal transplantation in a tuberous sclerosis patient with angiomyolipoma, renal carcinoma and life-threatening abdominal haemorrhages
}

\author{
Alf Corsenca ${ }^{1}$, Franziska Aebersold ${ }^{2}$, Holger Moch ${ }^{2}$, Patricia Bird ${ }^{3}$, Markus Weber ${ }^{4}$, \\ Günther Hofbauer ${ }^{5}$, Rudolf P. Wüthrich ${ }^{1}$ and Andreas L. Serra ${ }^{1}$ \\ ${ }^{1}$ Clinic for Nephrology, ${ }^{2}$ Institute of Surgical Pathology, Department of Pathology, ${ }^{3}$ Surgical Intensive Care Unit, \\ ${ }^{4}$ Department of Visceral and Transplant Surgery and ${ }^{5}$ Department of Dermatology, University Hospital, Zurich, \\ Switzerland
}

Keywords: abdominal haemorrhage; angiomyolipoma; renal carcinoma; renal transplantation; tuberous sclerosis complex

\section{Introduction}

Tuberous sclerosis complex (TSC) is an autosomal dominant disorder, with an incidence of approximately 1 in 6000. It arises from a genetic abnormality involving either the TSC1 gene on chromosome 9, or the TSC2 gene on chromosome 16. The protein product of the TSC1 gene is hamartin and that of TSC2 is tuberin. In cells, hamartin and tuberin form a complex, which inhibits the mammalian target of rapamycin (mTOR), a central controller of cell growth and proliferation [1].

Renal angiomyolipomas (AML) occur in $\sim 80 \%$ of patients with TSC. These lesions are composed of abnormal blood vessels, smooth muscle and fat cells and they have a tendency to bleed spontaneously, which can lead to life-threatening haemorrhagic shock [2]. Here we report on a patient with TSC suffering from recurrent severe haemorrhage from both kidneys due to extensive AML formation. Because of massive haemorrhage the patient was nephrectomized on the right side. One year later, the remaining left kidney was removed and a pre-emptive living renal transplantation was performed at the same time to prevent further renal haemorrhage.

Correspondence to: Andreas L. Serra, Clinic for Nephrology, University Hospital, CH-8091 Zurich, Switzerland.

Email: andreas.serra@usz.ch

\section{Case}

A 15-year-old, and till then a healthy young woman was evaluated in the year 2000 for abdominal pain after an injury which occurred while playing soccer. On clinical evaluation, small blisters (facial angiofibromas) were noted in her face and urine examination revealed microhaematuria. Kidney function was in the normal range and no proteinuria was detected. Computerized tomography (CT) scan of the abdomen showed enlargement of both kidneys with diffuse hypodense areas corresponding to multiple AMLs and isolated haematomas. A magnetic resonance imaging (MRI) scan revealed subependymal nodules in the brain. As the mother also showed facial angiofibromas and the brother was known to have diffuse bilateral AMLs in the kidneys, a clinical diagnosis of TSC was made. A genetic examination (Institute of Human Genetics, University Heidelberg, Germany) of the patient and her mother revealed a novel nonsense mutation (c. 408C $>\mathrm{A}, \mathrm{Y} 130 \mathrm{X}$ ) in the TSC2 gene, confirming the clinical diagnosis.

At the age of 17 years, the patient presented with a low haemoglobin level and a large spontaneous subcapsular haematoma was found in the left kidney (Figure 1A). This was treated conservatively but 1 year later, spontaneous bleeding recurred in the same kidney, with a drop in the haemoglobin concentration to $66 \mathrm{~g} / 1$. The estimated glomerular filtration rate (GFR) according to the Cockcroft-Gault formula was $78 \mathrm{ml} / \mathrm{min}$. An abdominal CT scan revealed persistence of the extensive subcapsular haematoma in the left kidney. A catheter embolization of the left upper pole artery was performed to stop bleeding. Despite arterial embolization, repeated spontaneous bleeding occurred in the left kidney, necessitating numerous blood transfusions to correct anaemia in the following 2 years. 

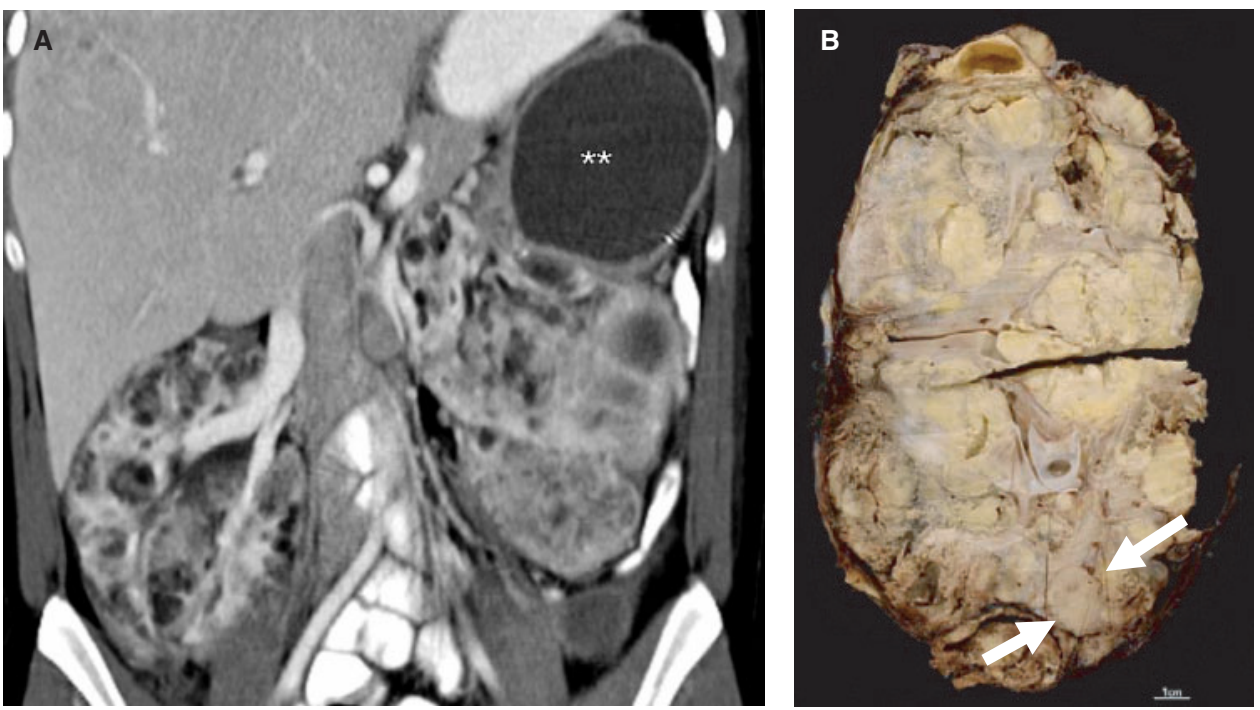

Fig. 1. Renal Manifestations of TSC. (A) CT scan of the abdomen in the year 2002, which shows large kidneys with multiple angiomyolipomas and a subcapsular haematoma (double asterisk) in the left kidney. (B) Macroscopic view of the left kidney after nephrectomy; co-existence of RCC (arrows) and angiomyolipomatous tissue.

In January 2005, bleeding occurred for the first time in the right kidney, causing a profound drop of the haemoglobin level. The serum creatinine increased to $210 \mu \mathrm{mol} / 1$ (MDRD GFR $28 \mathrm{ml} / \mathrm{min}$ ). A transarterial right kidney embolization was performed to stop the bleeding. During embolization extensive retroperitoneal bleeding occurred and the patient was admitted to the intensive care unit (ICU) for haemodynamic and respiratory stabilization. In the following days, the patient developed an abdominal compartment syndrome, sepsis and acute respiratory distress syndrome (ARDS). Kidney function declined rapidly and the patient became anuric. After 28 days in the ICU, an abdominal CT scan identified gas in the right-sided haematoma. An infection with anaerobic bacteria was suspected and the haematoma was evacuated and the right kidney removed. Following this intervention, clinical improvement occurred quickly and the patient was discharged with an estimated GFR (MDRD formula) of $34 \mathrm{ml} / \mathrm{min}$. Thirty-three units of packed red blood cells and 14 units of fresh frozen plasma (FFP) were transfused during the stay in the ICU to stabilize cardiovascular function.

Kidney function subsequently remained stable and the patient felt well, except for intermittent left lower back pain. Since the patient lived in a remote area and since there was a high risk for a new life-threatening bleeding in the left kidney, a decision was made to remove the remaining left kidney and to perform a living kidney transplantation from her 50-year-old healthy aunt.

The post-transplant course was uneventful and renal allograft function was excellent with a standard immunosuppressive regime consisting of tacrolimus, mycophenolate mofetil and prednisone. An allograft biopsy performed 3 months after transplantation showed no signs of rejection or calcineurin inhibitor toxicity. It has been reported that treatment with mTOR inhibitors has a favourable effect on extrarenal manifestation of TSC. MRI scan of the cortex revealed several subependymal nodules and cortical tubers with a maximal diameter of $10 \mathrm{~mm}$ in our patient. Subependymal giant cell tumours (SGCTs) arise from pre-existing subependymal nodules and can show massive haemorrhage, rapid growth and malignant transformation in adult patients. In a preliminary clinical trial, rapamycin was associated with regression of SGCT and in one patient, regrowth of SGCT after interruption of rapamycin treatment [3]. These promising results encouraged us to switch the immunosuppression from tacrolimus to rapamycin, in an attempt to prevent neurological complications in our patient. An MRI scan of the brain 6 months into treatment with rapamycin showed no progression of the subependymal nodules and cortical tubers, suggesting that rapamycin stabilized disease progression. Furthermore, after switching to rapamycin, the MDRD GFR increased from 40 to $48 \mathrm{ml} / \mathrm{min}$, which is in line with the known beneficial effect of a calcineurin-free immunosuppression on graft function.

\section{Pathological examination of left kidney}

Macroscopic examination of the resected left kidney showed a replacement of virtually the entire renal tissue by a huge AML (Figure 1B). Careful microscopical examination of the kidney (diametre $15 \mathrm{~cm}$ ) unexpectedly revealed the presence of a renal cell carcinoma (RCC) within the AML (Figure 2A). This RCC was sharply demarcated with a diametre of $1.9 \mathrm{~cm}$. It was classified as stage pT1a cN0 cM0, Fuhrman grade III. The tumour consisted of epithelioid cells with granular cytoplasm and perinuclear halo (Figure 2B). Hale's colloidal iron staining was 

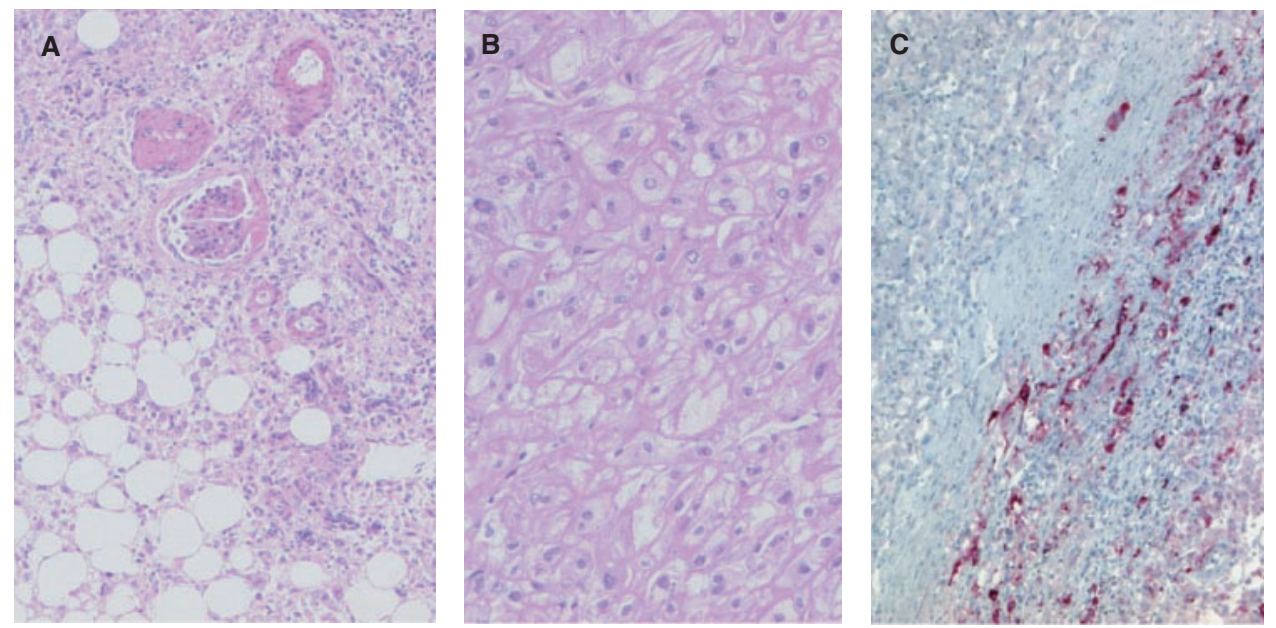

Fig. 2. Histology of renal AML and RCC. (A) The typical histomorphological characteristics of AML with smooth muscle and vessels (H \& E stain, original magnification $15 \times$ ). (B) histological presentation of the incidentally diagnosed RCC. Epithelioid tumour cells with granular cytoplasm (chromophobe-like) (original magnification $400 \times$ ). (C) HMB-45 expression of the AML (on the right) and lack of expression in the RCC (on the left) (original magnification $15 \times$ ).

only focally positive. Immunohistochemically, the RCC showed absence of HMB-45 and actin, whereas the surrounding AML was HMB-45 and actin positive (Figure 2C). The RCC also showed expression of vimentin and cytokeratin, but was negative for a RCC-specific antibody (RCC marker) and epithelial membrane antigen (EMA). This unusual histology was neither characteristic for a clear cell RCC (granular cytoplasm) nor a chromophobe RCC (vimentin expression). Therefore, this RCC could not be definitively classified.

\section{Discussion}

Here we report on a case of a young patient with TSC and severe bleeding from AML in both kidneys, who was nephrectomized and pre-emptively transplanted with success.

Renal pathologies in patients with TSC include AML, renal cysts and epithelial neoplasms [2]. Renal AML are fat-containing tumours, which are associated with a strong risk of haemorrhage due to the presence of abnormal vessels and pseudoaneurysms. AML occur in up to $80 \%$ of patients with TSC and are the cause of substantial morbidity. AML associated with TSC tend to be larger, bilateral, multifocal and present at a younger age compared with sporadic forms [4]. They can be diagnosed from a characteristic radiological pattern with the demonstration of fatty tissue in CT or MRI scans. A novel mutation, $\mathrm{C}>\mathrm{A}$ transversion at c.408 in exon 4 of TSC2 (Y130X) was found in the patient and her mother. This nonsense mutation will truncate the gene product of TSC2. Compared with TSC1, mutations in TSC2 are known to cause a more severe clinical phenotype, which is consistent with our case [5]. Thus, in our patient, renal AMLs with a diameter of $>4 \mathrm{~cm}$ were found in both kidneys, a finding which is very rare or not seen at all in TSC1 patients.

The risk of spontaneous bleeding is roughly correlated with the size of the AML, though there is substantial variation [6]. In our patient, the risk for the recurrence of life-threatening bleeding was very high. The remaining left kidney caused repetitive serious bleeding episodes originating from numerous large AML, some of them with a diameter $>4 \mathrm{~cm}$. AML more than $4 \mathrm{~cm}$ in diameter are associated with a high risk for symptomatic bleeding.

The therapeutic options to prevent bleeding from renal AML are limited. It has been suggested that large AML need to be surgically reduced or resected, or should be managed by catheter embolization to deprive the AML of its blood supply [7]. Bleeding complications from angiographic and surgical interventions often necessitate the removal of the entire kidney to control bleeding, as also occurred in our patient. Retroperitoneal bleeding is a well-known complication of AML, sometimes leading to death ('Wunderlich syndrome'). The nephrectomy of both kidneys represents a definitive treatment, but it implies subsequent haemodialysis treatment.

$\mathrm{Up}$ to now, 38 renal transplantations have been reported in TSC patients. In general patient outcome was good, with functioning allografts in 32 of 38 patients with a follow-up of up to 14 years [8]. It has been recommended that renal transplantation should be accompanied by nephrectomy of both kidneys, to remove the risk of malignant transformation and tumour growth associated with immunosuppression. Given the high risk for a recurrence of serious renal bleeding, the psychological burden for the patient and the family and the availability of a living kidney donor, the decision was made for our patient to remove the remaining left kidney, in combination with a living renal transplantation. 
The occurrence of RCC is a rare but well-known complication of TSC, that occurs in $1-2 \%$ of patients [2]. However, some of the previously reported RCC in tuberous sclerosis were later diagnosed as epithelioid AML, suggesting that the incidence of tuberous sclerosis-associated RCC is lower than some have suggested. Epithelioid AML represent an unusual subtype of AML, which is characterized by proliferation of predominantly epithelioid cells. In contrast to the triphasic (classic) AML, epithelioid AML can show a malignant behaviour. These tumours have a striking resemblance to RCC and often have high-grade histological features. Recently, it was shown that AML are associated with melanosome-associated protein (HMB-45) immunoreactivity, and that the HMB-45 expression is useful in differential diagnosis between AML and other tumours [9]. In our case, negative HMB-45 immunoreactivity was diagnostic for an RCC. The co-existence of AML and renal epithelial neoplasia is uncommon, with slightly more than 50 reported cases in the literature [10]. Pathological analysis of these case reports is difficult, because most have been published as clinical cases, lacking a detailed pathological description or exact histological typing. In addition, most of these cases were published before epithelioid AML was recognized as a separate entity. Few of the cases reported as clear-cell RCC were later reclassified as epithelioid AML.

According to the literature, in sporadic cases as well as in the setting of tuberous sclerosis, the most common renal cell neoplasm is clear-cell RCC. In patients with tuberous sclerosis, there is also a high frequency of oncocytomas. In a previous study, a large number of chromophobe-like RCC was detected in patients with tuberous sclerosis. Similarly, in previous reported cases in the literature of co-existing AMLs and renal epithelial neoplasia, an unusually high incidence of oncocytomas associated with AMLs is also seen $(12 \%)$. The RCC in our patient could not be assigned to one of the $2004 \mathrm{WHO}$ categories. Also in our case, the tumour showed chromophobe-like features. The granular cytoplasm was suggestive of a chromophobe-like RCC, but the vimentin expression excluded this diagnosis. All previously reported cases of co-existent AMLs and renal cell neoplasia in patients with tuberous sclerosis have been oncocytomas, or were of the clear-cell RCC, chromophobe-like or unclassified type.

The TSC1-TSC2 protein complex tunnels signals from growth factors, cell cycle and nutrients to regulate the activity of the downstream kinases mTOR, p70S kinase, 4E-BP1 and ribosomal S6 proteins. In tumour cells of TSC patients the mTOR pathway is aberrantly activated. In a mouse model for TSC, tumour size in the kidneys regressed with the treatment of the mTOR-specific inhibitor rapamycin [11]. Recent studies in humans demonstrated regression of renal AMLs during the administration of rapamycin to a patient with TSC and a regrowth when rapamycin was withdrawn [12]. Rapamycin is therefore a potential therapeutic agent and clinical trials of rapamycin in patients with TSC and AML are ongoing. Because of the history of repetitive renal bleeding necessitating nephrectomy, our patient would not have qualified for these clinical trials.

In summary, in patients with TSC, large renal AML can cause life-threatening abdominal bleeding. Combined nephrectomy and living renal transplantation removes the risk of severe bleeding, and represents definitive treatment in our patient.

Conflict of interest statement. None declared.

\section{References}

1. Tee AR, Fingar DC, Manning BD, Kwiatkowski DJ, Cantley LC, Blenis J. Tuberous sclerosis complex-1 and -2 gene products function together to inhibit mammalian target of rapamycin (mTOR)-mediated downstream signaling. Proc Natl Acad Sci USA 2002; 99: 13571-13576

2. Rakowski SK, Winterkorn EB, Paul E, Steele DJ, Halpern EF, Thiele EA. Renal manifestations of tuberous sclerosis complex: incidence, prognosis, and predictive factors. Kidney Int 2006; 70: $1777-1782$

3. Franz DN, Leonard J, Tudor C et al. Rapamycin causes regression of astrocytomas in tuberous sclerosis complex. Ann Neurol 2006; 59: 490-498

4. Nelson CP, Sanda MG. Contemporary diagnosis and management of renal angiomyolipoma. J Urol 2002; 168: 1315-1325

5. Au KS, Williams AT, Roach ES et al. Genotype/phenotype correlation in 325 individuals referred for a diagnosis of tuberous sclerosis complex in the United States. Genet Med 2007; 9: 88-100

6. Yamakado K, Tanaka N, Nakagawa $\mathrm{T}$, Kobayashi $\mathrm{S}$, Yanagawa M, Takeda K. Renal angiomyolipoma: relationships between tumor size, aneurysm formation, and rupture. Radiology 2002; 225: 78-82

7. Harabayashi T, Shinohara N, Katano H, Nonomura K, Shimizu T, Koyanagi T. Management of renal angiomyolipomas associated with tuberous sclerosis complex. J Urol 2004; 171: $102-105$

8. Schillinger F, Montagnac R. Chronic renal failure and its treatment in tuberous sclerosis. Nephrol Dial Transplant 1996; 11: $481-485$

9. Jungbluth AA, Iversen $\mathrm{K}$, Coplan $\mathrm{K}$ et al. Expression of melanocyte-associated markers gp-100 and Melan-A/MART-1 in angiomyolipomas. An immunohistochemical and rt-PCR analysis. Virchows Arch 1999; 434: 429-435

10. Jimenez RE, Eble JN, Reuter VE et al. Concurrent angiomyolipoma and renal cell neoplasia: a study of 36 cases. Mod Pathol 2001; 14: 157-163

11. Lee L, Sudentas P, Donohue B, Asrican K et al. Efficacy of a rapamycin analog (CCI-779) and IFN-gamma in tuberous sclerosis mouse models. Genes Chromosomes Cancer 2005; 42: 213-227

12. Wienecke R, Fackler I, Linsenmaier U, Mayer K, Licht T, Kretzler M. Antitumoral activity of rapamycin in renal angiomyolipoma associated with tuberous sclerosis complex. Am J Kidney Dis 2006; 48: e27-e29 